\title{
CRISPR takes genetic screens forward
}

\author{
Applying the molecular scissors to animals at scale is helping researchers delve into the potential roles of \\ different genes.
}

\section{Ellen P. Neff}

$\Lambda$ scribing function to a particular gene has been the goal of countless labswhether focused on understanding the pathology of, and treatments for, specific diseases or with broader, more basic developmental or evolutionary goals in mind. Disrupt a gene in a cell or an organism and the resulting phenotype (often, but not always) can help reveal much about that gene's purpose in both health when intact and in disease when mutated in a deleterious way. There are however many genes to consider. The Human Genome Project, completed in 2003, put the number of protein-coding genes in you and I at over 20,000 (the exact number remains up for debate). Counts in model organisms vary by species but also range in the tens of thousands. Moving one-by-one can be fruitful... but tedious.

A quicker option has long been found in genetic screening. Perturbing developing animal embryos or populations of cells, be it with a chemical such as ENU or other means such as radiation, can yield large numbers of mutants; a phenotype of interest can then be identified for study in closer detail, with genetic sequencing to determine the exact genetic change that occurred. But such screens are essentially random. More targeted technologies have become available, but with varying efficacy.

The arrival of CRISPR/Cas9 technology is improving the odds. "We have all these genomes, but we don't know what so many of these genes actually do," says Jason Yu at the Francis Crick Institute in the UK. Screening with CRISPR, he says, "is a way to start moving through those in a higher throughput fashion - to identify what those genes are doing and what the consequences are when a gene is mutated."

Researchers tout the simplicity and efficacy of these 'molecular scissors' in perturbing genomes at scale to discover those mutants of interest-all in an unbiased fashion. "We design an experiment that really asks biology to tell us what the most important factors that are contributing to a certain process are," says Robert Manguso of the Broad Institute and Massachusetts General Hospital in Boston. Studied in isolation, a particular gene you think should

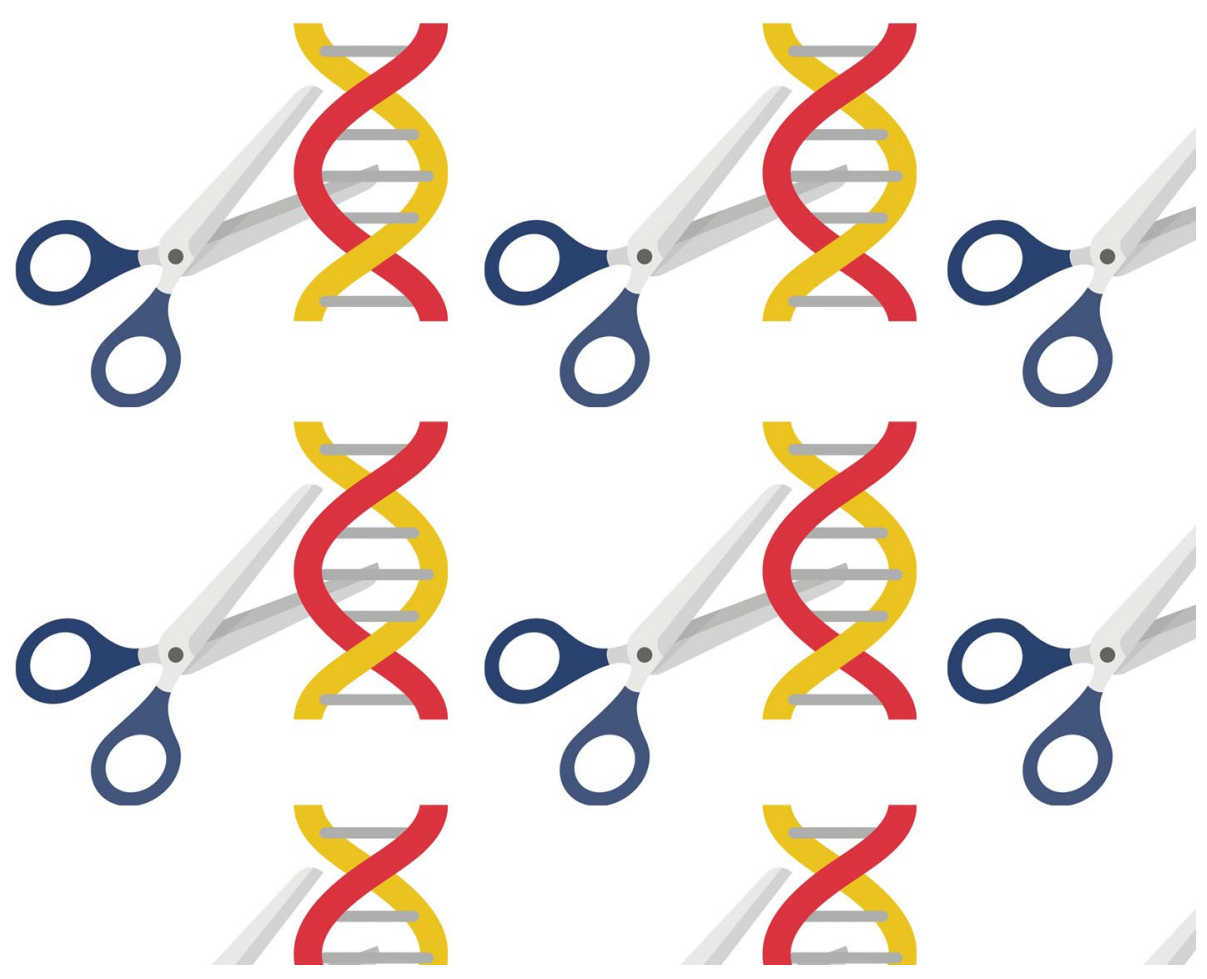

Cut (and cut and cut some more) CRISPR/Cas9 is helping research increase the throughput of genetic screening. Credit: Anatolii Riabokon / Alamy Stock Vector

be important might in fact have a smaller role than realized; screening can rank the likely importance of dozens, hundreds, and even thousands of genes simultaneously. Getting a 'hit' improves your starting point for more detailed study, he says.

CRISPR screening is currently at work in vitro and in vivo in various models and organisms, but adapting it to a given model comes with different challenges and caveats.

\section{Into the pool}

There are three general components to any genetic screen: the perturbation, the model, and the assay ${ }^{1}$. CRISPR has been shaking up the perturbation element. The system involves two components: the DNAcutting enzyme Cas9 and a guide RNA to a particular place in a gene. When Cas9 finds the sequence that matches the guide's instructions, it creates a double stranded break in the DNA; that break is then mended, albeit imprecisely, leaving behind a mutation that (more often than not) can disrupt the function of the gene affected.

"As a basic research tool, CRISPR is not fundamentally different from its predecessor," says John Doench of the Broad Institute, but it has been proving more specific and more versatile. Pre-CRISPR, RNAi was all the rage for a time, he says, but RNAi approaches could suffer from offtarget affects, and its means of interference in the genome meant genes were only knocked down in function-not out entirely. CRISPR is not without limitations but it seems to work better, at least in screening contexts.

It can be relatively straightforward for a lab to deploy as well. Cas9 is commercially available, as are guide RNAs that can be purchased in large libraries. You don't 
necessarily need the largest library you can buy though, notes $\mathrm{Yu}$ - it can sometimes be more appropriate to use more targeted subsets. For those with smaller, more targeted gene lists, the desired sequences can also be designed and synthesized in the lab with the help of numerous online tools.

Screening pools of mutagenized cells offers genome-wide scale. These are an effective means to test thousands and thousands of genes at a time for how they might function in a given cell, as well as when and where, says Doench. "In mammals, we have hundreds if not thousands of unique cell types, and genes can do different things at different times and places."

For such pooled CRISPR screens, Cas9 and libraries of guide RNAs introduce mutations to cells in culture; each cell in the pool will then carry a single gene knockout. Together, the pools can be screened for a particular phenotype of interest, such as survival or growth under different experimental conditions. Sequencing the 'hits'-those with interesting phenotypes - can reveal and rank the candidate genes likely to be involved.

Take the identity of the receptor for murine norovirus, says Doench. Researchers had been studying the murine version of the nasty stomach bug for years, but its receptor in the mouse was proving elusive. Doench and collaborators used a pooled CRISPR approach in vitro to knock out every gene in the mouse genome in search of the receptor that mediates viral entry ${ }^{2}$; in vivo follow up with a knockout mouse validated the finding from the initial screen.

Getting the CRISPR tools in at the start can sometimes present a challenge though. "You need to deliver a large protein into mammalian cells that did not evolve to necessarily express this protein, and this protein did not evolve to function in a mammalian cell," says Doench. In the case of human and murine cell lines, lentiviruses carrying the Cas9 and guides have been the vehicle of choice; these retroviruses can integrate with the mammalian genome to introduce permanent changes.

Interest isn't just limited to mammalian models; the technology has recently been applied to Drosophila melanogaster cell lines too. Raghuvir Viswanatha, a postdoc in Norbert Perrimon's lab at Harvard was intrigued by the arrival of CRISPR but uncertain about its fly cell applications. "I had no expectations that it would work at all," he says. That's because the fly field lacks an established lentivirus equivalent; instead, he and his colleagues turned to bacterial site-specific recombination via plasmids to

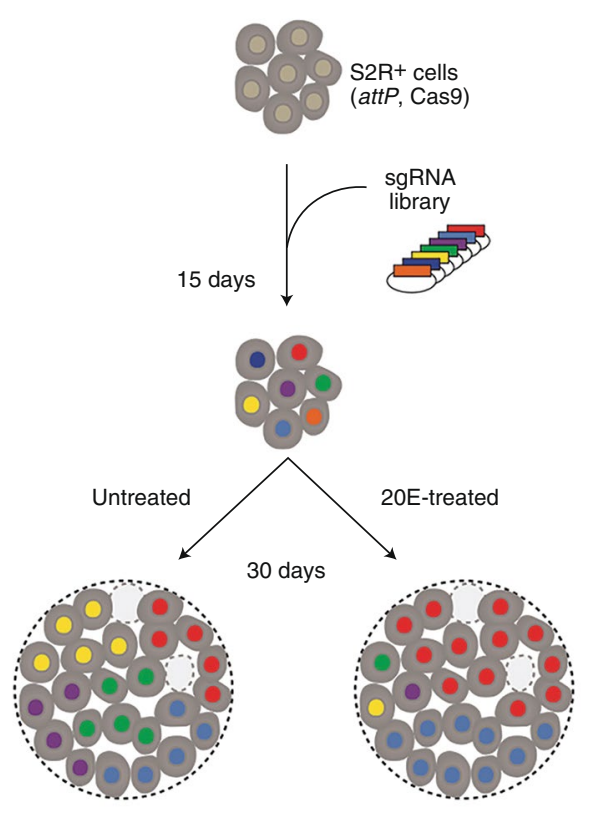

Pooled together | Schematic of Viswanatha and colleague's pooled screening approach in Drosophila cells. Reprinted with permission from Okamoto $2018^{5}$ (Elsevier).

transfect their cell line with Cas9 and the guide RNAs desired ${ }^{3}$.

Why bother trying to apply pooled screening in fly cells when murine and human cell line options are readily available? Flies share conserved proteins and functions with mammals but their genomes generally have less redundancy to sort through, says Viswanatha-where humans might have two genes controlling a function, a fly will have only one. In their proof-of-concept paper, Viswanatha and his colleagues screened for fly fitness genes, identifying many that had yet to be studied in any detail ${ }^{4}$. A second paper combined results from a pooled CRISPR screen in the Perrimon lab with a more traditional in vivo approach from Naoki Yamanaka's lab at the University of California Riverside. Both groups had converged on a gene that mediates reception of a molting hormone called ecdysone ${ }^{5}$, but the in vivo approach involved creating and then assaying numerous RNAi-mutated flies. The CRISPR screen found the hit in an unbiased fashion and with fewer false positives to sort through and validate, says Viswanatha.

\section{Context clues}

Cell lines can indeed offer the advantage of scale, but they inherently lack some biological context. Cells grown in dishes live relatively pampered lives, says Manguso. Nutrients are replenished regularly, there's ample oxygen, and the cells face no influence from a larger immune system or contact with competing cells nearby. In vitro, they can just grow as they please, with caretakers around to keep them happy. In a body, life isn't so simple. But a tumor, for example, can thrive in the harsh in vivo microenvironment all the same. How cancerous cells adapt to such challenges could yield therapeutic targets to stop them, says Manguso. Particularly among cancer researchers, he notes a growing recognition that cells need to be studied in the context in which researchers care about them.

Manguso and his lab study cancer, with a focus on improving immunotherapies. This, they believe, requires an intact immune system-something that can only be found in a completely immunocompetent, wildtype animal. When CRISPR arrived on the scene, he started using it in mouse cancer cell lines; meanwhile, others at the Broad were developing large CRISPR screening libraries. A question emerged: would it be possible to introduce a CRISPR library to murine cancer cells in vitro and then transplant those mutated cells into mice?

"A lot of people thought that going in vivo was going to be a complete waste of time," Manguso recalls, "It would be really impossible to see a true signal." The results are indeed a bit noisier than working with cells in culture, but the approach has been useful for identifying targets to enhance immunotherapies nonetheless.

In his first in vivo screening paper, Manguso and his colleagues zeroed in on a phosphatase that, when deleted, increased the efficacy of immunotherapies in the mice $^{6}$. Another revealed that when the ADAR1 gene, which produces an enzyme that negatively regulates the sensing of double stranded RNA, is deleted, tumor cells will start to behave as if they are infected with a virus ${ }^{7}$ - this leads to cures in almost $100 \%$ of animals. "This gene turned out to work in a way in tumors that we just would not have put together if we hadn't seen it come out of a screen," says Manguso. "I can confidently say we would not have been studying if it had not come out of one of these early screens."

Transplanting pools of mutated cells into a mouse does come with challenges. "When you're screening cells in a dish, you can take them out, look at them under the microscope, and count them. Once you put them into an animal, it's a black furry box," says Doench. Replicates are important, as is quantifying the cells responsible for the phenotype. Unlike in vitro studies where you can add as many flasks of cells needed to screen through thousands of cells, animals have limits. Manguso notes that out of a million mutated cells, about 100,000 will 


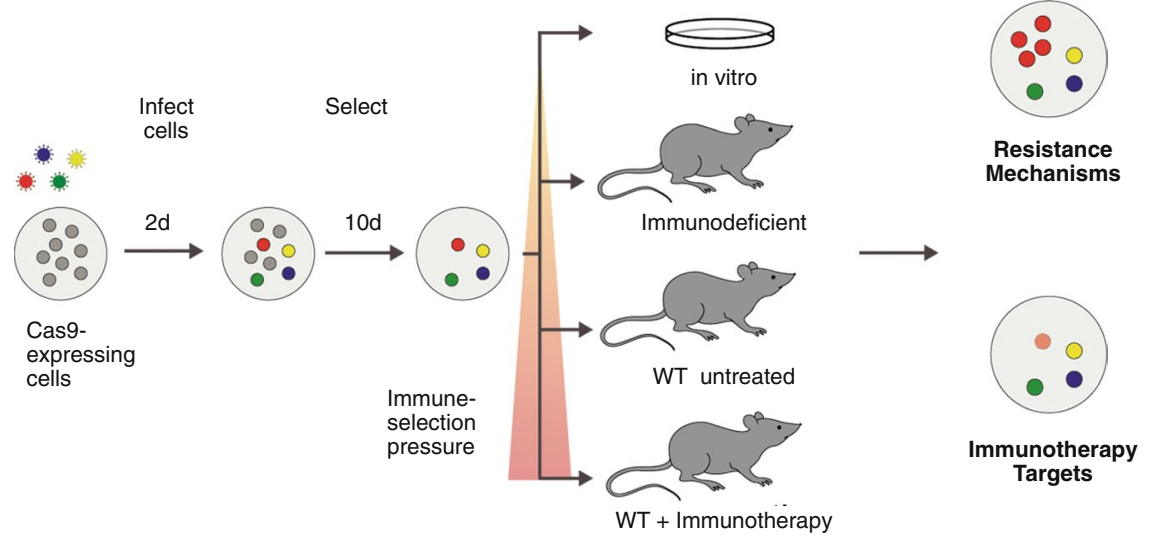

Add some immunity | Schematic of Manguso and colleague's approach to mutating tumors with CRISPR for transplant in vivo. Credit: R. Manguso

successfully engraft into a mouse; that limits the size of the guide library and also means more replicate animals are required for a given experiment. The animals themselves can experience secondary effects associated with tumors that need to be monitored for, and at the end of an experiment they must be sacrificed so that the cells can be retrieved and analyzed to determine which mutations fared better or worse under the selective pressure of the immunotherapy applied.

Nevertheless, Manguso considers those challenges worth it and is working with others to make the approach more readily available to researchers outside the Broad. "The field that has benefited the most from pooled genetic screening is cancer biology," says Manguso. "It's pretty obvious what the right result is, because we want the cancer cells to die." But for any field where an intact organism is an important consideration-be it immunology more broadly, or growing areas such as understanding the host-gut microbiome-CRISPR offers a fruitful means to screen through a lot of genes with the larger in vivo context in mind.

\section{Mutants from cell one}

Keep the spine but lose the fur and you'll find a useful feature: large embryos, and lots of them. Those of the highly fecund zebrafish, Danio rerio, and African clawed frogs, Xenopus tropicalis and Xenopus laevis, start off life as a single cell resting a top a large yolk mass. At that single cell stage, easily visible under the microscope, researchers can add all the machinery necessary for CRISPR/Cas9 to operate directly within the growing embryo itself. The externally developing animals can then be monitored in real time for changes as they grow with the given mutations. Such endogenous editing doesn't lend itself to genome-wide screens as conveniently as pooled screens originating in vitrothere just isn't enough material in a living animal-but CRISPR is nonetheless changing the way researchers look for phenotypes in whole organisms, to better understand normal development and how it can go awry as well as to look for phenotypes that might recapitulate a particular human disease.

The zebrafish has long offered many mutant options for researchers to study, thanks to forward genetics and concerted efforts to generate stable lines of numerous chemically mutagenized fish, such the Tübingen Screen in the late 90s. "Of course it's very powerful," says Adam Miller at the University of Oregon, "The organism is telling you what's important to it." But such screening can be tedious, in terms of both the husbandry involved to handle the many fish needed to and the task of mapping the actual genetic mutation involved. That's not to mention the fact that there's no control over the actual genes targeted; some genes can remain unaffected by chemicals or radiation.

Prior to CRISPR, several methods had emerged to create targeted mutations in both zebrafish and Xenopus, including the use of morpholinos, zinc finger nucleases, and TALENs. But each came with caveatsoff-target effects, expense, and the time needed to create them-that can be limits to their use for high-throughput screens, an advantage for many to using zebrafish and Xenopus models. CRISPR is turning out to be both simple and effective.

After the first paper was published demonstrating CRISPR editing in zebrafish, Miller and technician Arish Shah, both then working in the lab of Cecilia Moens at the Fred Hutchinson Cancer Research Center in Seattle, wanted to see whether they could apply the technology for screening purposes. Cas9 was indeed straightforward in the fish. "The baseline function of Cas9, which is to find a DNA target and then cut it, works great in fish and we didn't really need to do any modifications," says Shah. Optimizing concentrations of the enzyme and guide RNAs took some tweaking-too much RNA can be toxic to the embryos-but they ultimately developed a means to target multiple genes in parallel with combinations of different guide RNAs ${ }^{8}$.

Since then, Christian Mosimann's lab has demonstrated that injected Cas9 protein, rather than the RNA to make it, also suffices ${ }^{9}$. Others have since published additional methods - Roland $\mathrm{Wu}$ and colleagues at the University of California San Francisco moved injection of Cas9-guide RNA complexes from that single zebrafish cell to the slightly larger and more accessible yolk ${ }^{10}$, while Engin Deniz and others at Yale have detailed the steps for a screening approach in Xenopus tropicalis ${ }^{11}$.

Because the screens aren't genomewide-not technically impossible, though it would be decidedly time-consuming to individually inject enough embryos to work through the whole genome, says Miller-the guides used are designed to target particular sets of genes of interest. That list can come from forward genetics or prior studies, or from genes implicated in human diseases that have orthologues in the animals. Wu is looking for cardiac mutations in the fish, while Deniz screens his frogs to find tadpoles that match various developmental defects he observes in his pediatric patients. Miller is looking for mutations involved in the development of electrical synapses in

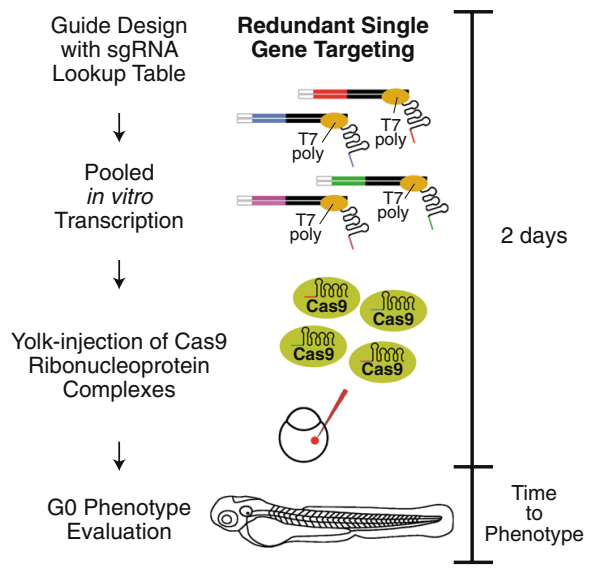

Embryo editing | Schematic of Wu and colleague's method for using CRISPR technology to screen generation 0 zebrafish. Reprinted with permission from Wu $2018^{10}$ (Elsevier). 


\section{Box 1 | Mosaicism as the goal}

In some cases, mosaicism can mean false positives or that certain phenotypes might be missed. But in others, that mosaicism can be a valuable feature.

Serine Avagyan, a MD/PhD in Leonard Zon's lab at Harvard, studies clonal hematopoiesis of indeterminate potential, or CHIP. Over time, she explains, stem cell clones can accumulate mutations. Should a particularly advantageous mutation emerge, the clones that divide from that mutated cell can end up outcompeting other populations with different genetic makeups. CHIP arises from a single mutation and has been proposed to be one of the initial steps in the formation of blood-based cancers, such as leukemia. It is a challenge however to collect blood mutation data from people over long periods of time, she says; as such, knowledge of CHIP comes from a single data point, lacking information about prior and subsequent mutations.

When CRISPR is applied endogenously to mice to create stable lines, a given mutation will be present in all cells

zebrafish-he's set up his own lab to look more closely at several genes that emerged from those initial screens in Seattle. He's added single cell sequencing and proteomics methods to his labs' repertoire to better refine their starting list for future screens.

In the fish and the frogs, researchers can screen hundreds of mutagenized individuals for phenotypes in first-generation animals right off the bat, but the animals are mosaics-some cells are mutated, others remain wild-type (Box 1). "You have to take a phenotype with a grain of salt," says Miller, "You have a bunch of different cells with different genetic make ups coming together in complex ways." Even though the machinery is injected at cell one, that cell starts to divide quickly. Rates vary over development but as a result, CRISPR will mutate some cells before they divide while missing others.

Screening is just the beginning.

\section{Following up...}

Regardless of the model used-pooled cells, transplanted tumors, or endogenously edited fish or frogs-the researchers interviewed for this story all emphasized that a CRISPR screen is only a first step towards understanding the function of a particular gene. "You never really understand how something works until you of a given tissue, Avagyan says; some researchers instead mutate different cells and then transplant those to a mouse to see how competition plays out, but such transplants don't inherently reflect the environment in which CHIP develops in humans. Hematopoiesis, meanwhile, is conserved between mammals and, in the case of CRISPR, mosaically mutant fish.

Avagyan and colleagues have combined CRISPR/Cas9 with a technique called 'Zebrabow'13 that labels blood stem and progenitor cells with different colored fluorescent proteins. Embryos are injected with various combinations of guide RNAs that introduce mutations in genes associated with human blood cancers. The fish are then left to grow up. The researchers sequence the mutant clones from blood samples at different time points and, as the stems cells are colorfully labeled, they visually can follow the 'winning' clone over time. "You can see the [endogenous] competition right in front of you," says Avagyan, across hundreds of developing fish.

break it," says Manguso, but the screens are only hypothesis generating-you need still need to validate and determine the actual mechanisms involved.

"You get excited about the phenotypewith a note of caution-and then go ahead and take those mutations forward through the germline and study them in just classic ways that geneticists have always studied these sorts of things," says Miller. CRISPR can help at both phases, says Shah: reagents used in the screen that produce an interesting phenotype can then be used to generate a stable mutant line for that mutation-a faster and easier proposition than making mutant lines for all the starting candidates on a list.

There remain questions about what a screen may miss. At the screening stage you need to be willing to sacrifice specificity for high sensitivity, says $\mathrm{Wu}$ - there may be false positives and phenotypes that just don't penetrate enough to be picked up. Screens also tend to turn up cell autonomous phenotypes, notes Manguso. Perturbing a gene that contributes to extracellular effects are less likely to be detectable, especially in contexts where neighboring cells lack the particular mutation and can thus pick up the slack.

Compensation might also need to be accounted for as well. A recent paper revealed that the way CRISPR knocks out genes can turn up compensating genes through a process known as nonsense mediated decay ${ }^{12}$. "That was something that we weren't aware of when we started out," says Miller. "As you're developing a screen you need to pay attention to the fact that by knocking out a particular gene, you might actually be upregulating related genes that might then obscure your phenotype of interest."

Growing pains, but CRISPR-and its coming iterations-are nevertheless likely here to stay. "I really think the reason it works so well in vivo is because CRISPR technology actually works much, much better than previous technologies," says Manguso. As CRISPR/Cas technology continues to improve, it may one day do it all-knock genes out, knock them down, and knock them in. Different Cas enzymes could yield combinatorial experiments in which multiple genes are knocked out at the same time. And the prospect of using base editors to introduce increasingly precise mutations to a gene is growing on the horizon as well.

For all the promise of the technology to help researchers screen through myriad genes, there's a critical underlying point: all must begin with good models and assays, says Doench. "The right answer has to come from the biology that you want to study first," he says. "CRISPR is great-it's a really wonderful tool-but it's not magic fairy dust. If you don't have a good model or you don't have a good assay, the fact that you're using CRISPR is not going to help you."

CRISPR wisely.

\section{Ellen P. Neff}

Lab Animal.

e-mail: Ellen.neff@us.nature.com

Published online: 18 December 2019

https://doi.org/10.1038/s41684-019-0445-0

References

1. Doench, J. G. Nat. Rev. Genet. 19, 67-80 (2018).

2. Orchard, R. C. et al. Science 353, 933-936 (2016).

3. Viswanatha, R. et al. Curr. Protoc. Mol. Biol. https://doi. org/10.1002/cpmb.111 (2019).

4. Viswanatha, R., Li, Z., Hu, Y. \& Perrimon, N. eLife 7, e36333 (2018).

5. Okamato, N. et al. Dev. Cell 47, 294-305.e7 (2018).

6. Manguso, R. T. et al. Nature 547, 413-418 (2017).

7. Ishizuka, J. J. et al. Nature 565, 43-48 (2019).

8. Shah, A. N., Davey, C. F., Whitebirch, A. C., Miller, A. C. \&

Moens, C. B. Nat. Method 735, 535-540 (2015).

9. Burger, A. et al. Development 143, 2025-2037 (2016).

10. Wu., R. S. et al. Dev. Cell 46, 112-125.e4 (2018).

11. Deniz, E., Mis, E.K., Lane, M. and Khokha, M. K. pp 163-174 In: Vleminckx K. (eds) Xenopus. Methods in Molecular Biology, vol 1865. (Humana Press, New York, NY, 2018).

12. El-Brolosy, M. A. et al. Nature 568, 193-197 (2019).

13. Henninger, J. et al. Nat Cell Biol. 19, 17-27 (2017). 